\title{
Sugammadex in the Management of Sinus Tachycardia after Rocuronium Administration: A Case Report
}

\author{
Eduardo Fernandes Orioli Guimarães, Muriel Mofreita Saldanha, Tiago Coelho Fortes, \\ Marcelo Grisolia, Marcos Lopes de Miranda, Carlos Darcy Alves Bersot
}

Department of Anesthesia, Lagoa Federal Hospital, Rio de Janeiro, Brazil

Email: carlosbersot@gmail.com

Received 8 July 2014; revised 9 August 2014; accepted 30 August 2014

Copyright (C) 2014 by authors and Scientific Research Publishing Inc.

This work is licensed under the Creative Commons Attribution International License (CC BY).

http://creativecommons.org/licenses/by/4.0/

(c) (i)

\begin{abstract}
In rare cases, rocuronium has been associated with dose-related tachycardia, probably by a cardiac muscarinic M2 receptor blockade mechanism. We report the case of a 30-year-old female who underwent excision of a branchial cyst under general anesthesia. This patient presented an episode of sinus tachycardia $(130 \mathrm{bpm})$ shortly after anesthesia induction with propofol, sufentanyl, and rocuronium. Tachycardia could not be explained by any cause other than the use of rocuronium, which was reverted with sugammadex. Two minutes after sugammadex administration, heart rate normalized, corroborating our hypothesis that rocuronium induced the sinus tachycardia observed in our patient. The patient recovered well from the anesthetic-surgical procedure and showed no further cardiovascular, ventilatory, or neurological changes, being transferred to the post-anesthesia care unit, and finally discharged to the ward.
\end{abstract}

\section{Keywords}

Rocuronium, Sugammadex, Anesthesia, Sinus Tachycardia

\section{Introduction}

The introduction of neuromuscular blocking agents (NBA) to anesthetic practice allowed great advance in airway management and helped to optimize the surgical field by inhibiting spontaneous ventilation and causing relaxation of skeletal muscles. Rocuronium is an aminosteroid non-depolarizing NBA with rapid onset and intermediate duration of action. Its use in anesthesia is increasing due to the possibility of its application in rapid sequence endotracheal intubation and due to existence of an effective reverser. Despite being quite safe, in rare 
cases rocuronium has been associated with dose-related tachycardia, which may be related to interactions of this steroidal NBA with cardiac muscarinic M2 receptors [1] [2]. We report a case of sinus tachycardia following anesthesia induction in which tachycardia could not be explained by any cause other than the use of rocuronium. Interestingly, heart rate normalized after administration of sugammadex, a modified gamma-cyclodextrin indicated to reverse neuromuscular blockade produced by rocuronium.

\section{Case Report}

A 30-year-old female (height $1.55 \mathrm{~m}$, weight $58 \mathrm{~kg}$, ASAI) was admitted to our hospital for excision of a branchial cyst under general anesthesia. During preanesthetic visit the patient denied previous anesthesias, allergies, and regular use of medications. Her preoperative tests were within normal limits.

Patient was not premedicated, but on admission in operating room she had no signs of anxiety. She was monitored with standard monitors (5-lead electrocardiogram, non-invasive blood pressure, pulse oximetry, and expiratory capnography; Infinity Vista XL monitor, Drager, Lubeck, Germany), bispectral index (BIS; A-2000 BIS Monitoring System, Aspect Medical Systems, Newton, USA), and neuromuscular transmission monitor (TOF-Watch SX, Organon Ireland, Dublin, Ireland). Baseline heart rate, arterial blood pressure, and pulse oximetry were within normal limits. Two peripheral venous access $(20 \mathrm{G})$ were established in upper limbs, one exclusively for propofol infusion.

After preoxygenation with $100 \%$ oxygen, anesthesia was induced and maintained by target-controlled infusion of propofol PFS 1\% (plasma concentration $3.0 \mathrm{mcg} \cdot \mathrm{mL}^{-1}$; Diprifusor, AstraZeneca, Alderley Park, UK). After loss of consciousness, verified by no response to verbal stimulation and a BIS value of 32, sufentanyl 35 mcg and rocuronium bromide $35 \mathrm{mg}$ were administered as bolus injections.

Heart rate increased (130 bpm; sinus rhythm) shortly after rocuronium administration, while arterial blood pressure remained stable (mean arterial pressure was around $60 \mathrm{mmHg}$ ). Considering the possibility of an awake patient, a bolus dose of $3 \mathrm{mg}$ midazolam was injected, despite a BIS value of 28. As the patient maintained sinus tachycardia with heart rate around $125 \mathrm{bpm}$, we proceeded to successfully and uneventfully orotracheal intubation and tried to rule out possible causes of sinus tachycardia. After initiation of controlled mechanical ventilation (Fabius GS, Drager, Lubeck, Germany), pulmonary auscultation, end-tidal carbon dioxide $\left(\mathrm{ETCO}_{2}\right)$ value, and capnography waveform were normal and the patient had no cutaneous rash or any other sign or symptom of anaphylaxis. Additionally, the patient had BIS values within the range for general anesthesia $(<45$ throughout the episode) and no signs or symptoms of awareness. At that moment, besides an elevated heart rate, only train-of-four (TOF) monitoring was presenting an inappropriate value: we observed an abnormal TOF response after rocuronium administration, with maintenance of one or two counts in response to stimulation.

After ruling out anaphylactic reaction, pain, awareness, and other common causes of tachycardia in this scenario, we thought that an atypical and rare response to rocuronium could be related to the sinus tachycardia, and proceeded to reversal of neuromuscular blockade with $200 \mathrm{mg}$ of sugammadex. About two minutes after sugammadex bolus, we observed a decrease in heart rate from 120 to $76 \mathrm{bpm}$ (sinus rhythm) and a TOF value of $108 \%$.

As the patient had neither arterial blood pressure instability nor ventilatory changes during the tachycardia episode, we chose to continue with the anesthesia. After injection of a bolus dose of $4 \mathrm{mg}$ of cisatracurium, TOF monitoring presented a fatigue pattern followed by no response to stimulation. The surgery was performed without any further complications. At the end of the procedure, patient was extubated and transferred to post-anesthesia care unit (PACU) where she maintained stability of both cardiovascular and respiratory functions, and had no signs of cognitive dysfunction. After one hour in PACU, she was discharged to the ward with an Aldrete score of 10 .

\section{Discussion}

In the pase, important hemodynamic changes were commonly associated with D-tubocurarine and gallamine, some of the earliest NBAs used in clinical practice. The synthesis of the contemporaneous NBAs, such as the aminosteroids pancuronium, vecuronium, and rocuronium, and the benzylisoquinolines atracurium and cisatracurium, decreased the incidence of adverse cardiovascular events, but did not eliminate them. Anaphylactic reactions and non-immune histamine release are commonly involved in hemodynamic changes. Additionally, interactions with receptors other than the nicotinic receptor, such as muscarinic receptors (especially cardiac M2 
subtype), may cause undesirable cardiovascular changes. NBAs have different degrees of affinity for muscarinic receptors. Gallamine is among those with highest affinity, having tachycardia due to cardiac muscarinic receptors blocking as one of its known side effects [3]. A similar response is observed with the use of pancuronium. Conversely, Appadu et al. [2] have demonstrated that rocuronium has the lowest affinity for cardiac muscarinic receptors among aminosteroids [2]. In this way, cases of tachycardia due to cardiac muscarinic receptors blocking are rare with rocuronium and usually associated with higher doses (dose-related effect) [1]. However, with the increasing use of this NBA in clinical practice, rare events will be increasingly observed. Indeed, in a recent study of Sorensen et al., $10 \%$ of patients in the rocuronium group $\left(1.0 \mathrm{mg} \cdot \mathrm{kg}^{-1}\right)$ showed tachycardia (heart rate $>100 \mathrm{bpm}$ ) after anesthesia induction [4].

In our case, tachycardia could not be explained by any cause other than the use of rocuronium. It has already been demonstrated that rocuronium does not induce histamine release [5], eliminating non-immune histamine release as an explanation for the tachycardia episode. A high incidence of rocuronium-induced anaphylactic reactions has been reported by French and Norwegian studies [6]. However that was not corroborated by further studies from other parts of the world. Nowadays, it is accepted that the incidence of anaphylactic reactions associated with rocuronium is similar to that of other NBA [6]. Our patient had no cutaneous, respiratory, or arterial blood pressure changes throughout the anesthetic and surgical procedures, and later in the PACU. Thus, the patient presented only one of the diagnostic criteria for anaphylactic reaction (tachycardia) [7], which makes anaphylactic reaction diagnosis improbable. It is important to note that we have not proceeded to further laboratorial investigations such as quantification of tryptase levels or cutaneous provocative tests because these tests frequently give false results in situations like ours [7]. Neuromuscular blocking in an awake patient is very uncomfortable and provokes autonomic alterations, notably tachycardia. This possibility was ruled out after the administration of a bolus dose of midazolam (with no change in heart rate). Additionally, the patient had BIS values within the range for general anesthesia throughout the tachycardia episode and no signs or symptoms of awareness.

Based on recent papers that showed successful resolution of rocuronium-induced anaphylactic reactions with sugammadex administration [8] [9] and considering that this drug encapsulates rocuronium molecules and prevents its action on nicotinic receptors, we administered sugammadex expecting reversal of the suspected muscarinic receptors blocking. Interestingly, tachycardia resolved after two minutes of sugammadex administration, which is the same time necessary for neuromuscular blocking reversal, supporting the hypothesis of rocuronium-induced tachycardia. Thus, after ruling out common differential diagnosis possibilities and considering the straight temporal correlation between the initiation of the tachycardia episode and rocuronium injection and the immediate decrease of heart rate after sugammadex administration, we hypothesized that rocuronium induced the sinus tachycardia observed in our patient, probably by a cardiac muscarinic M2 receptor blockade mechanism. Of note, rocuronium was not used in high doses, which made our case even more uncommon.

\section{References}

[1] Stevens, J.B., Hexker, R.B., Talbot, J.C. and Walker, S.C. (1997) The Haemodynamic Effects of Rocuronium and Vecuronium Are Different under Balanced Anaesthesia. Acta Anaesthesiologica Scandinavica, 41, 502-505. http://dx.doi.org/10.1111/j.1399-6576.1997.tb04731.x

[2] Appadu, B.L. and Lammbert, D.G. (1994) Studies on the Interaction of Steroidal Neuromuscular Blocking Drugs with Cardiac Muscarinic Receptors. British Journal of Anaesthesia, 72, 86-88. http://dx.doi.org/10.1093/bja/72.1.86

[3] Dunlap, J. and Brown, J.H. (1983) Heterogeneity of Binding Sites on Cardiac Muscarinic Receptors Induced by Neuromuscular Blocking Agents Gallamine and Pancuronium. Molecular Pharmacology, 24, 15-22.

[4] Sorensen, M.K., Bretlau, C., Gatke, M.R., Sorensen, A.M. and Rasmussen, L.S. (2012) Rapid Sequence Induction and Intubation with Rocuronium-Sugammadex Compared with Succinylcholine: A Randomized Trial. British Journal of Anaesthesia, 108, 682-689. http://dx.doi.org/10.1093/bja/aer503

[5] Levy, J.H., Davis, G.K., Duggan, J. and Szlam, F. (1994) Determination of the Hemodynamics and Histamine Release of Rocuronium (Org 9426) When Administered in Increased Doses under N2O/O2-Sufentanil Anesthesia. Anesthesia \& Analgesia, 78, 318-321. http://dx.doi.org/10.1213/00000539-199402000-00020

[6] Bhananker, S.M., O’Donnell, J.T., Salemi, J.R. and Bishop, M.J. (2005) The Risk of Anaphylactic Reactions to Rocuronium in the United States Is Comparable to That of Vecuronium: An Analysis of Food and Drug Administration Reporting of Adverse Events. Anesthesia \& Analgesia, 101, 819-822.

http://dx.doi.org/10.1213/01.ANE.0000175213.87556.D8 
[7] Simons, F.E., Ardusso, L.R., Bilò, M.B., El-Gamal, Y.M., Leford, D.K., Ring, J., Sanchez-Borges, M., Senna, G.E., Sheikh, A. and Thong, B.Y. (2011) World Allergy Organization Guidelines for the Assessment and Management of Anaphylaxis. World Allergy Organization Journal, 4, 13-37. http://dx.doi.org/10.1097/WOX.0b013e318211496c

[8] McDonnell, N.J., Pavy, T.J., Green, L.K. and Platt, P.R. (2011) Sugammadex in the Management of Rocuronium-Induced Anaphylaxis. British Journal of Anaesthesia, 106, 199-201. http://dx.doi.org/10.1093/bja/aeq366

[9] Kawano, T., Tamura, T., Hamaguchi, M., Yatabe, T., Yamashita, K. and Yokoyama, M. (2012) Successful Management of Rocuronium-Induced Anaphylactic Reactions with Sugammadex: A Case Report. Journal of Clinical Anesthesia, 24, 62-64. http://dx.doi.org/10.1016/j.jclinane.2011.04.015 
Scientific Research Publishing (SCIRP) is one of the largest Open Access journal publishers. It is currently publishing more than 200 open access, online, peer-reviewed journals covering a wide range of academic disciplines. SCIRP serves the worldwide academic communities and contributes to the progress and application of science with its publication.

Other selected journals from SCIRP are listed as below. Submit your manuscript to us via either submit@scirp.org or Online Submission Portal.
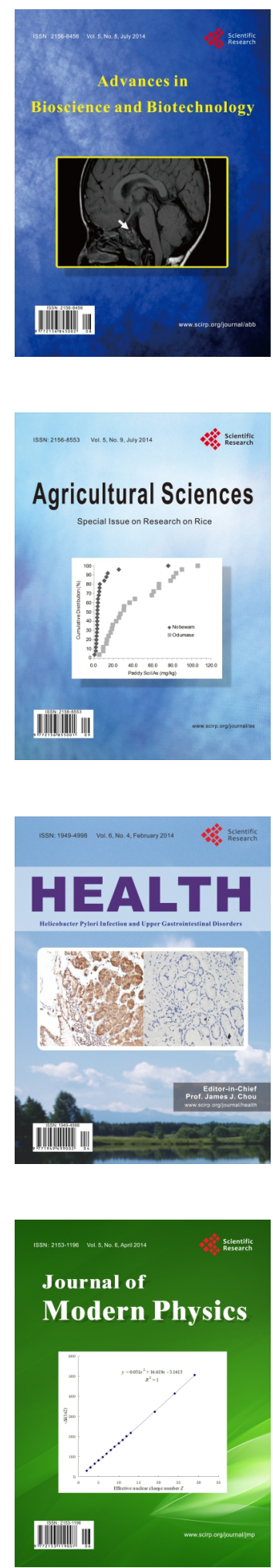
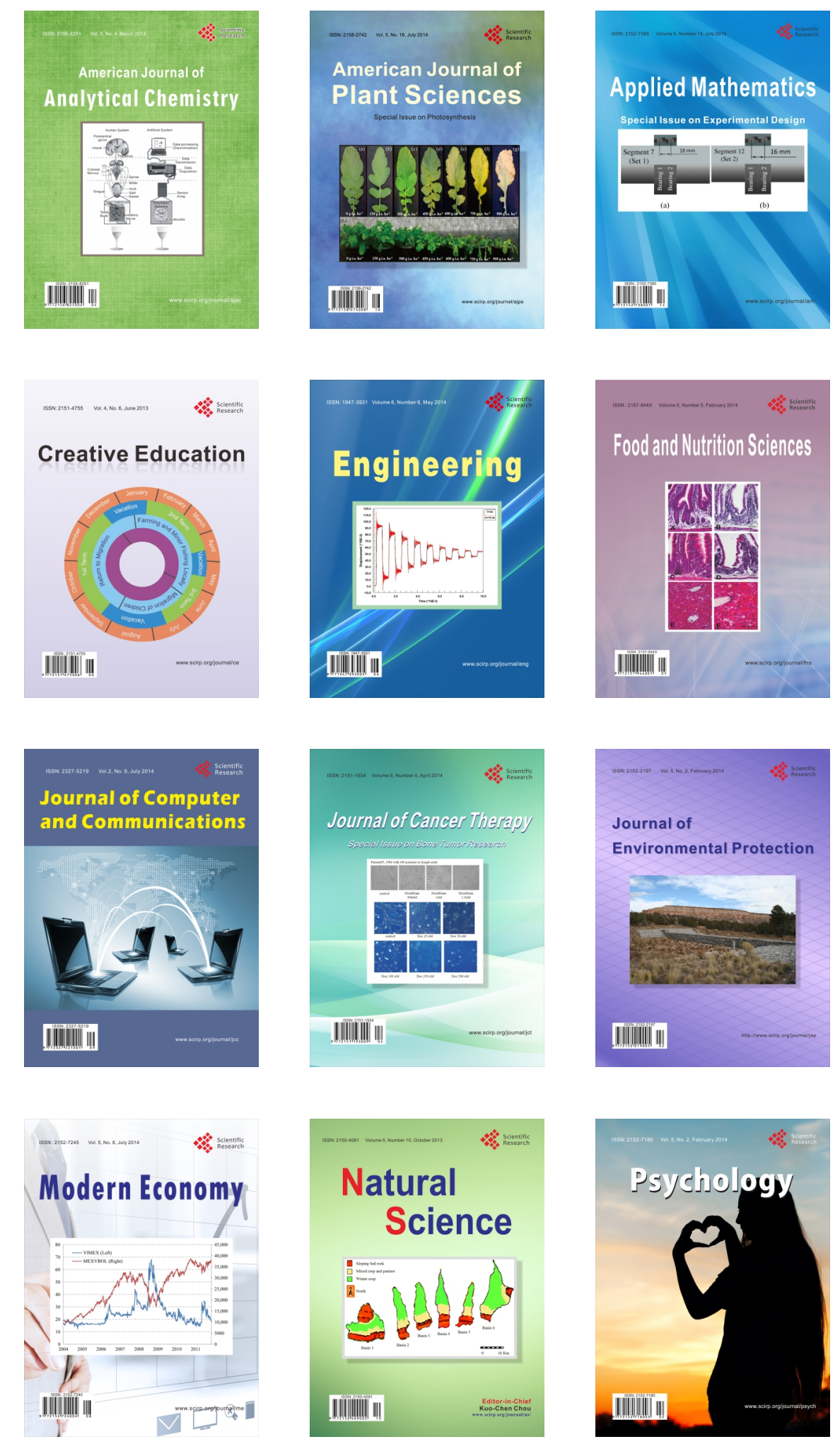